\title{
Influence of Alternating and Static Magnetic Fields on Drug Release from Hybrid Hydrogels Containing Magnetic Nanoparticles
}

\author{
Marianna Uva1,2, Daniela Pasqui ${ }^{1,2}$, Lorenzo Mencuccini1 ${ }^{1,2}$, Serena Fedi ${ }^{1,2}$, \\ Rolando Barbucci1,2* \\ ${ }^{1}$ Department of Biotechnology, Chemistry and Pharmacy, University of Siena, Via Aldo Moro 2, Siena, Italy \\ ${ }^{2}$ Interuniversity Research Centre for Advanced Medical Systems (C.R.I.S.M.A.), Viale Giacomo Matteotti, Colle \\ di Val d'Elsa, Siena, Italy \\ Email: ${ }^{*}$ rolando.barbucci@unisi.it
}

Received 28 February 2014; revised 29 March 2014; accepted 17 April 2014

Copyright (C) 2014 by authors and Scientific Research Publishing Inc.

This work is licensed under the Creative Commons Attribution International License (CC BY). http://creativecommons.org/licenses/by/4.0/

(c) (i) Open Access

\section{Abstract}

Hybrid hydrogels of carboxymethylcellulose (CMC), containing two different amounts of $\mathrm{CoFe}_{2} \mathrm{O}_{4}$ magnetic nanoparticles $(50 \%$ and $70 \%$ in relation to the quantity of the polymer) as crosslinkers, were prepared. The hybrid hydrogels were chemically and morphologically characterized and their viscoelastic properties and swelling degrees were analyzed. The hydrogels were tested as controlled drug delivery systems by applying one static and two different alternating magnetic fields. The application of the two alternating magnetic fields (AMF) to the hybrid hydrogels induced a higher release of methylene blue (MB), used as a model drug, than without the application of any magnetic field, especially at low frequency $(4 \mathrm{~Hz})$ and high magnetic intensity $(0.5 \mathrm{~T})$. In contrast, when the hybrid hydrogels were exposed to a static magnetic field (SMF) the release of MB was slowed down. Furthermore the two different amounts of magnetic nanoparticles induce different responses to the magnetic field. The greater number of nanoparticles in the CMC-NP-70 hydrogel leads to the formation of some NPs clusters limiting the drug release; conversely, the CMCNP-50 hydrogel, containing a lower amount of nanoparticles, shows a higher release of MB vs. time. In conclusion, we were able to get a potential system for modulation of the drug delivery: the release behaviour of hybrid hydrogels can be modulated by applying alternating and static magnetic fields cyclically. A possible explanation for the release mechanism is about the structural modification of the polymeric chains that occurs when the hybrid hydrogels are exposed to the magnetic fields.

\footnotetext{
${ }^{*}$ Corresponding author.
}

How to cite this paper: Uva, M., et al. (2014) Influence of Alternating and Static Magnetic Fields on Drug Release from Hybrid Hydrogels Containing Magnetic Nanoparticles. Journal of Biomaterials and Nanobiotechnology, 5, 116-127. 


\section{Keywords}

\section{Magnetic Hydrogels, Alternating Magnetic Field, Static Magnetic Field, Controlled Release, Cobalt Ferrite Nanoparticles}

\section{Introduction}

Magnetic gels, also called ferrogels, consist of polymer networks into which magnetic nanoparticles are embedded. The increasing interest in this class of biomaterial is due to their particular properties, which derive from complex interplay between the mechanical and chemical properties of the hydrogel matrix and the magnetic behaviour of the entrapped magnetic nanoparticles (NPs). The possibility of controlling the ferrogel using an externally applied field may give rise to its application in medicine, as a controlled drug release system [1]-[4]. The external application of magnetic fields represents an alternative to other stimuli-responsive methods, such as changing temperature or $\mathrm{pH}$. External magnetic stimuli are mainly used for controlled drug release and represent one of the most common strategies in materials designed for biomedical devices. The literature contains many reports on the development of hydrogels containing magnetic metal oxide NPs. The NPs are incorporated into the polymer matrix by mixing them with a pre-synthesized gel, or by adding them during the gel formation reaction [5]-[7]. In both cases the NPs are physically embedded within the hydrogel; as a consequence they may be released from the hydrogel or change their position when subjected to a magnetic field [8]. In either event a substantial modification of the properties takes place, which in some cases may render them completely unreliable for applications in the biomedical field.

In previous papers we illustrated the synthesis of polysaccharide based hydrogels containing $\mathrm{CoFe}_{2} \mathrm{O}_{4} \mathrm{NPs}_{\text {as }}$ crosslinkers of the macromolecules (carboxymethylcellulose and hyaluronic acid). These novel magnetic hybrid hydrogels were chemically, morphologically and mechanically characterized [9]-[11]. In this paper we exploit the magnetic carboxymethylcellulose hybrid hydrogels as systems for the controlled release of a model drug, i.e. methylene blue (MB), under the application of two alternating magnetic fields, AMF (4 Hz, $0.5 \mathrm{~T}$ and $40 \mathrm{KHz}, 2$ $\mathrm{mT})$, and a static magnetic field, SMF (0.5 T). Moreover, the magnetic CMC based hydrogels containing different amounts of $\mathrm{CoFe}_{2} \mathrm{O}_{4} \mathrm{NPs}(50 \%$ and $70 \%$, in relation to the quantity of the polymer) were prepared in order to evaluate the different distribution of the NPs inside the hydrogel and their effect on the release of MB. Modulation of the drug release was also achieved, based on the application of AMF and SMF in sequence.

In light of the hydrogels' swelling behaviour when placed under a magnetic field, a possible interpretation of the mechanism of drug release from the CMC-NP hybrid hydrogels when subjected to static and alternating magnetic field is proposed.

\section{Materials and Methods}

\subsection{Materials}

The sodium salt of carboxymethylcellulose (CMC, average MW $700 \mathrm{kDa}$, with a degree of carboxymethylation equivalent to 0.86) was purchased from Sigma-Aldrich. Commercial aqueous dispersions of cobalt ferrite NPs were provided by Colorobbia (Italy). The NPs show the $\mathrm{CoFe}_{2} \mathrm{O}_{4}$ stoichiometry, and the nominal diameter of the primary NPs in water is about $20 \mathrm{~nm}$, according to the manufacturer's specifications. The silane coupling agent 3-aminopropyltrimethoxysilane (APTMS), N-hydroxysuccinimide (NHS), N-(3-dimethylaminopropyl)-N-ethylcarbodiimide hydrochloride (EDC), 1,3-diaminopropane (DAP), and all the reagents were purchased from Sigma-Aldrich.

\subsection{Synthesis of the Hydrogels}

The synthesis of the CMC polysaccharide based magnetic hydrogels containing cobalt ferrite NPs was performed according to the previously described procedure [9]. Briefly, $\mathrm{CoFe}_{2} \mathrm{O}_{4} \mathrm{NPs}$ were functionalized by APTMS with the aim of obtaining free amino groups on their surface, which were able to react with the carboxylic acid groups of the polysaccharide. The amount of NPs was tuned to two ratios: $50 \%$ and $70 \% \mathrm{w} / \mathrm{w}$, in relation to the 
amount of the polymer. The CMC based hydrogels containing 50\% w/w and $70 \% \mathrm{w} / \mathrm{w}$ of NPs are referred to as CMC-NP-50 and CMC-NP-70, respectively. The crosslinking reaction achieved with less than $50 \%$ NPs does not to lead the formation of a stable hydrogel. A further CMC hydrogel was prepared, using 1,3 diamminopropane (DAP) as a crosslinking agent and following the previously reported chemical route [12], and referred to as CMC-DAP. This hydrogel type was taken as reference material.

\subsection{Morphological Analyses}

FESEM (Field-Emission Scanning Electron Microscope) and EDX (Energy Dispersive X-ray) analyses were performed using a ZEISS FESEM SIGMA VP. The FESEM images were recorded using accelerating voltages, in a range from $1 \mathrm{kV}$ to $10 \mathrm{kV}$, and a beam current of $2.4 \mathrm{~A}$. EDX point-by-point mapping was performed using an Oxford Instruments EDX system/Oxford Instruments INCA connected to the FESEM, with an INCA X-sight detector at an accelerating voltage of $20 \mathrm{KV}$. Both hydrated and dried CMC-NP-50 and CMC-NP-70 hydrogels were analyzed.

Finally, hydrated CMC-NP 50 hydrogel samples were viewed by using cryo stage with the aim to investigate possible structure changes of the hydrogel under the application of AMF and SMF. Hydrated samples were transferred into the cryo-stage and observed at $-25^{\circ} \mathrm{C}$ by under partial vacuum.

\subsection{Potentiometric, Swelling and Rheological Measurements}

Potentiometric titrations of the hybrid hydrogels were carried out in aqueous solutions at $25^{\circ} \mathrm{C}$ using a TitraLab 90 equipment from Radiometer Analytical. Titrations were performed by stepwise additions of standard 0.068 $\mathrm{M} \mathrm{NaOH}$ at an addition rate of $40 \mu \mathrm{l}$ every five minutes, which gives sufficient time to reach equilibrium between the solution and the hydrogel. To avoid $\mathrm{CO}_{2}$ contamination the surface of the solution was kept under a nitrogen stream. The amount of free $\mathrm{COOH}$ groups in the hydrogel were calculated from the $\mathrm{NaOH}$ equivalents used.

The water uptake (WU), or swelling degree, was determined gravimetrically following the previously reported procedure [10]. Freeze dried samples (freeze dried hydrogels containing about $10 \%$ water) were immersed in $0.15 \mathrm{M} \mathrm{NaCl}$ at room temperature and stored for more than $24 \mathrm{~h}$ until they reached a swelling equilibrium. The WU\% was calculated using the following equation:

$$
\text { Water Uptake }=(\mathrm{Ws}-\mathrm{Wd}) / \mathrm{Wd} \times 100
$$

where Ws is the weight of the swollen hydrogel at equilibrium and Wd is the initial weight of the dried hydrogel.

Swelling measurements of the same magnetic hydrogels were also performed by applying an AMF and a SMF under the same conditions used for the methylene blue release.

Rheological measurements were performed on hydrogels at their maximum swollen state. An AR2000 controlled strain Rheometer (TA Instruments, Leatherhead, United Kingdom) was used in the parallel plate configuration at a controlled temperature of $25^{\circ} \mathrm{C} \pm 1{ }^{\circ} \mathrm{C}$. A dynamic stress sweep test was performed at a frequency of $1 \mathrm{~Hz}$. The test gives the values of the storage modulus (G') and the loss modulus (G”). The G' value provides information about the energy stored in the material during deformation stress while G" describes its viscous character.

\subsection{Methylene Blue Dye Loading and Release under Alternating and Static Magnetic Fields}

Freeze-dried hydrogels were soaked in a $4 \mathrm{ug} / \mathrm{ml}$ solution of methylene blue (MB) and left to swell to their maximum swelling degree. The amount of MB contained in the hydrogels was determined by calculating the difference between the initial feeding amount of MB and the amount of MB left in the solution after hydrogel soaking.

The release of $\mathrm{MB}$ was performed at room temperature in a $1 \mathrm{~cm}$ quartz cuvette for spectrophotometric measurements, containing $3 \mathrm{~mL}$ of $0.15 \mathrm{M} \mathrm{NaCl}$ solution. Exactly weighed amounts of freeze-dried CMC-NP hydrogels loaded with MB were soaked in the cuvette and the release of MB was tested under the effect of an alternating magnetic field (AMF) and a static magnetic field (SMF). The release of MB from the same hydrogel samples without the application of a magnetic stimulus was determined and used for reference. In order to verify 
whether the application of an external magnetic field had any effect on a non-magnetic hydrogel (i.e. without NPs), the same experiments were performed using a CMC-DAP hydrogel.

At determined experimental times, an exact volume of the solution was taken and the UV-spectra were recorded using a spectrophotometer (Lambda 650 UV/Vis Spectrophotometer, Perkin Elmer). The amount of MB contained in the solutions was calculated by a MB calibration curve, built by measuring the absorbance of the solution at a known concentration of MB at $\lambda=664 \mathrm{~nm}$. Two different pieces of equipment were used to generate the AMF:

1) two copper solenoids connected to two ferrite bars, in the centre of which the cuvette containing the magnetic hybrid hydrogel was placed. The equipment generated an AMF with a frequency of $40 \mathrm{kHz}$ and magnetic induction of $2 \mathrm{mT}$;

2) two permanent magnets placed on a proper support which, once connected to the electric power, rotated with a frequency of $4 \mathrm{~Hz}$. Between two magnets, where the cuvette containing the magnetic hydrogel was placed, there was an AMF of $0.5 \mathrm{~T}$.

The static magnetic field was generated by two permanent magnets with a length of $75 \mathrm{~mm}$, made of 15 magnets of $5 \mathrm{~mm}$ each. The magnetic field to which the sample is exposed depends on the distance between the sample and the magnets. The sample was placed at a distance of $1 \mathrm{~mm}$ to the magnets and was subjected to a SMF of $0.5 \mathrm{~T}$.

\section{Results and Discussion}

\subsection{Synthesis and Chemical Characterization of the Hydrogels}

As previously reported, synthesis of the hybrid hydrogels involves two steps [9]. The first step concerns the functionalization of the NPs with 3-aminopropyltrimethoxysilane (APTMS) to introduce $-\mathrm{NH}_{2}$ groups on the surface of the nanoparticles (NP-NH ). The second step involves the formation of amide bonds between the carboxylic groups of the CMC polymer and the primary amines of the $\mathrm{NPs}_{-} \mathrm{NH}_{2}$, as crosslinker. The synthesized hybrid hydrogels are CMC-NP-50 and CMC-NP-70 containing 50\% and 70\% w/w NPs/polymer, respectively. Figure 1 shows the chemical structure of the CMC-NP hydrogel.

Once formed, the hydrogels were washed several times. No release of NPs in the washing solutions was ever noticed.

Figure 2 reports FESEM images of hydrated and freeze-dried CMC magnetic hydrogels. The hydrated surface of the hydrogels (CMC-NP-50 and CMC-NP-70) appears smooth and free of bumps (Figure 2(A)). The presence of NPs was evident only after the hydrogel was freeze-dried and at high magnification. On the CMCNP-50 surface, NPs up to $100 \mathrm{~nm}$ (Figure 2(B)) are observed, while on the CMC-NP-70 there are clusters of NPs of about $2-3 \mu \mathrm{m}$ (Figure 2(C)).

Figure 3 shows the iron distribution on freeze-dried CMC-NP hydrogels. Figure 3(A) shows uniform iron distribution on CMC-NP-50, while Figure 3(B) illustrates inhomogeneous iron distribution on CMC-NP-70, confirming the formation of bigger NP clusters.

The crosslinking degree of the two hydrogels was determined by potentiometric titrations of the remaining free - $\mathrm{COOH}$ groups of the CMC polymer. The crosslinking degree calculated was $80 \%$ for CMC-NP-50 and $63 \%$

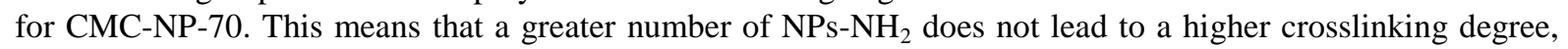
probably because of their tendency to form aggregates, due to their magnetic interaction. The water uptake (WU) of the two magnetic hydrogels is reported in Figure 4, together with that of the CMC-DAP hydrogel for comparison (67\% crosslinking degree). All hydrogels show a high water uptake, at more than 4000 times the weight of the freeze-dried hydrogel. These high WUs are mainly due to the strong hydrophilic nature of the CMC polymer, as well as to the formation of numerous highly hydrophilic amide groups in the crosslinking reaction [12]. The presence of NPs reduces the WU of the magnetic hydrogels when compared to CMC-DAP, but the different amount of NPs contained inside them does not affect the WU. The hydrogel containing the highest number of nanoparticles (CMC-NP-70) shows only a slightly lower WU than that containing 50\% w/w of NPs (CMCNP-50), perhaps due to the formation of a higher number of amide groups in the latter.

Table 1 reports the values of G' and G" of the two hybrid hydrogels compared to CMC-DAP. The frequency sweep test evidenced a "gel-like" behaviour for all the hydrogels, with G' being greater than G" [13]; this is further confirmation of the success of the crosslinking reaction. The G' of the hydrogel CMC-NP-70 is slightly less than that of CMC-NP-50. The aggregation of NPs in the CMC-NP-70 prevents the gel from presenting bet- 


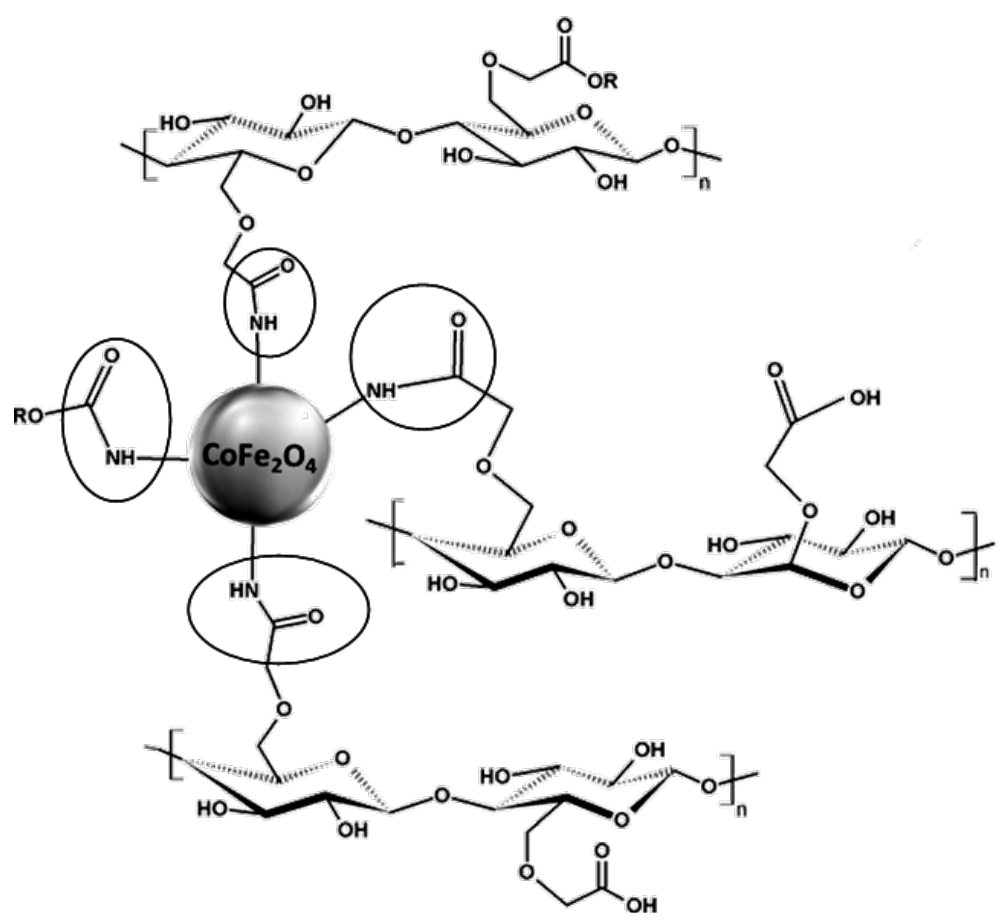

Figure 1. The chemical structure of the CMC-NP hydrogel.
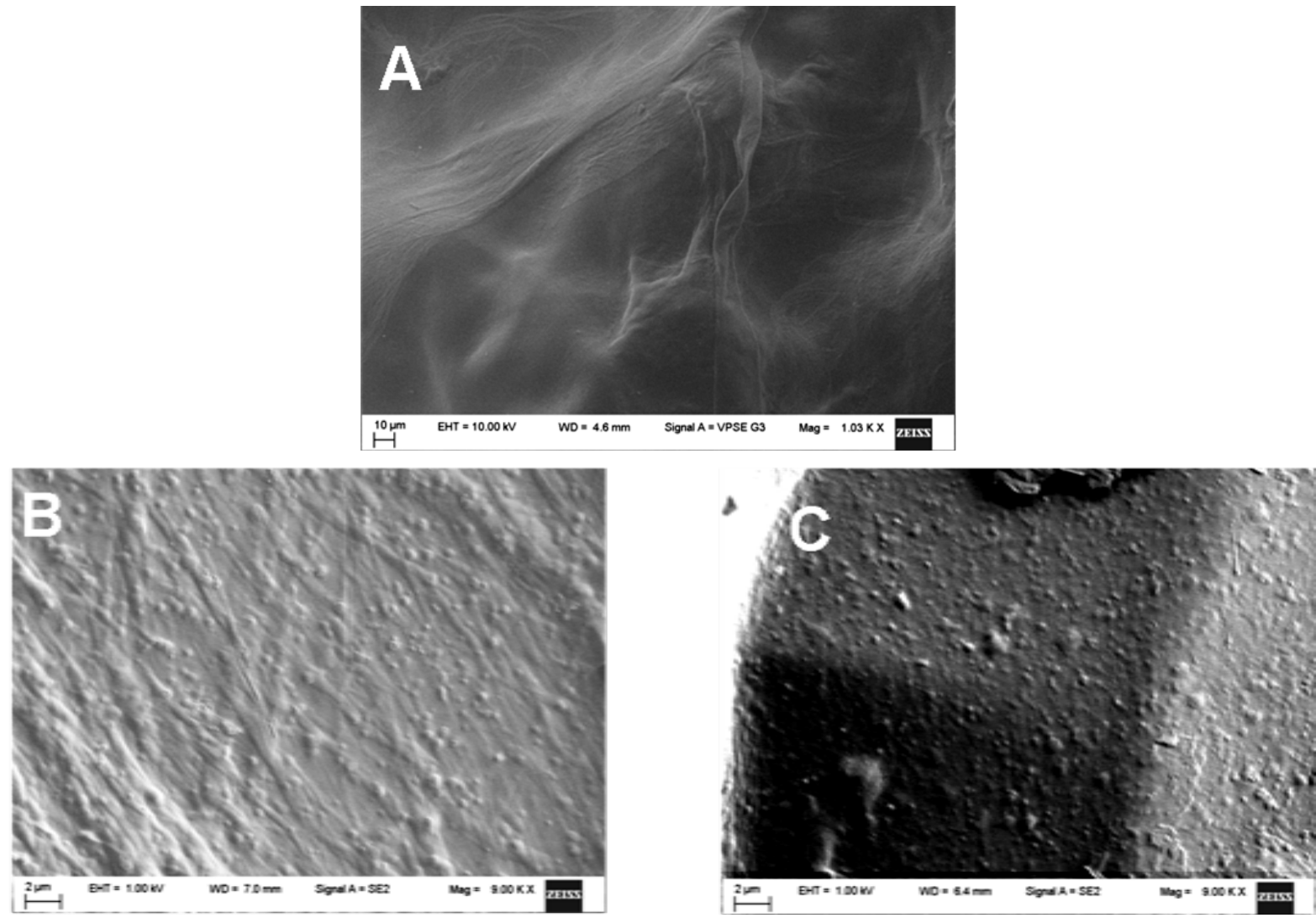

Figure 2. FESEM images of: (A) hydrated CMC-NP-50 and CMC-NP-70 hydrogels at a magnification of 1000×, (B) freeze-dried CMC-NP-50, and (C) freeze-dried CMC-NP-70 hydrogels, the latter two at a magnification of $9000 \times$; the circle highlights the presence of NP aggregates. 


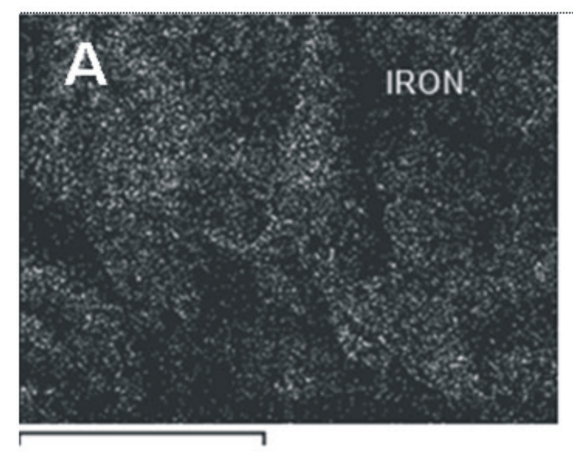

$600 \mu \mathrm{m}$

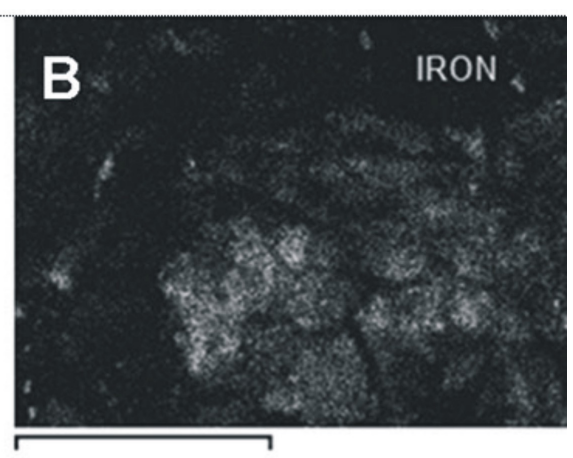

$600 \mu \mathrm{m}$

Figure 3. EDX maps showing iron distribution on (A) CMC-NP-50 and (B) CMC-NP-70.

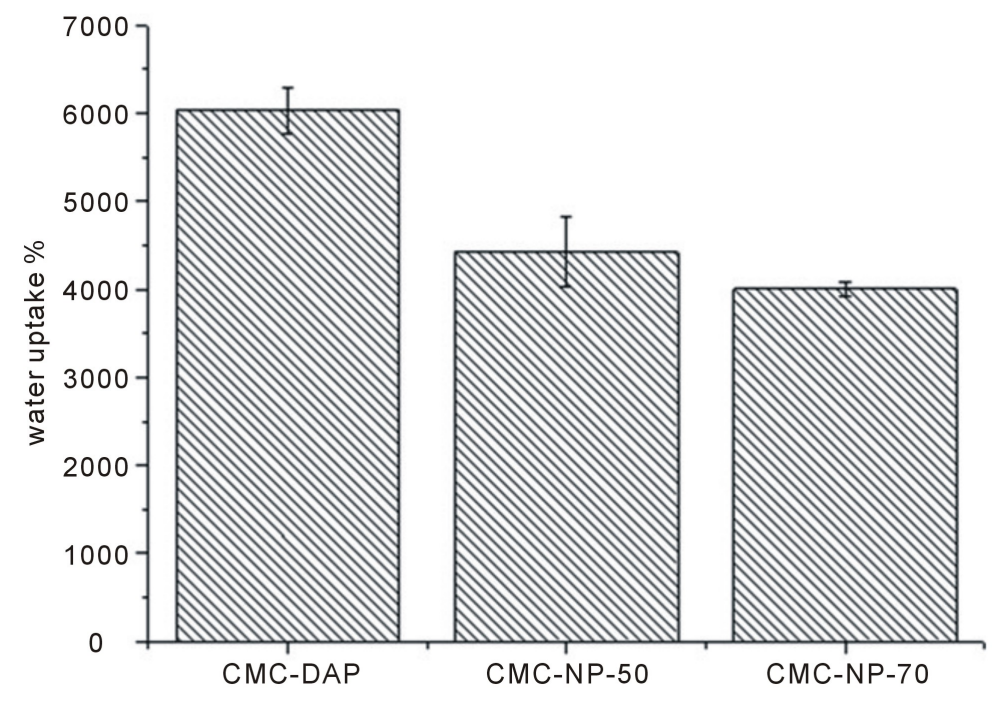

Figure 4. Water Uptake for magnetic and CMC-DAP hydrogels in $0.15 \mathrm{M}$ $\mathrm{NaCl}$ solution at their maximum swelling degree.

Table 1. G' and G” values for magnetic and CMC-DAP hydrogels.

\begin{tabular}{ccc}
\hline Sample & $\boldsymbol{G}^{\prime}(\mathbf{P a})$ & $\boldsymbol{G}^{\boldsymbol{\prime}}(\mathbf{P a})$ \\
\hline CMC-DAP & $500 \pm 50$ & $60 \pm 30$ \\
CMC-NP-50 & $3300 \pm 300$ & $90 \pm 20$ \\
CMC-NP-70 & $2000 \pm 200$ & $110 \pm 20$
\end{tabular}

ter mechanical properties.

In conclusion, the presence of NPs reduces the water uptake in comparison to CMC-DAP, which shows a similar crosslinking degree (67\%), and increases mechanical characteristics of the magnetic hydrogels.

\subsection{Release Behaviour of the Hybrid Hydrogels}

CMC-NP-50, CMC-NP-70 and CMC-DAP were loaded with methylene blue (MB) dye as a model drug. The amount of $\mathrm{MB}$ absorbed at equilibrium was similar in the three types of hydrogel $(0.011 \mu \mathrm{g} \mathrm{MB} / \mu \mathrm{g}$ in freezedried CMC-NP-50, $0.014 \mu \mathrm{g} \mathrm{MB} / \mu \mathrm{g}$ in freeze-dried CMC-NP-70, and $0.012 \mu \mathrm{g} \mathrm{MB} / \mu \mathrm{g}$ in freeze-dried CMCDAP). The release trend for the two hybrid hydrogels (CMC-NP-50 and CMC-NP-70) was similar taking into account experimental error, reaching a plateau of 56\% of MB released after 120 minutes (see Figure 5). 


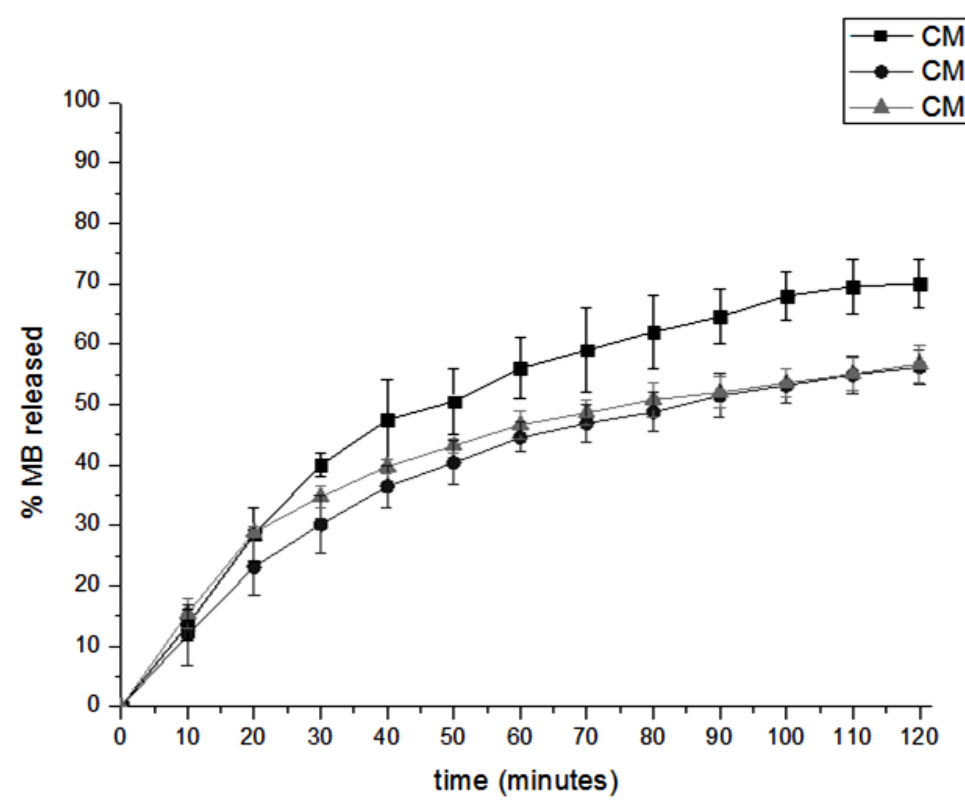

Figure 5. Release curve of methylene blue from CMC-DAP (black squares), CMCNP-50 (dark grey circles) and CMC-NP-70 (grey triangles).

This agrees with the water uptake values for these two hydrogels, which are also similar. The number of nanoparticles contained in the hydrogels did not affect the release trend. In contrast, the amount of MB released from the CMC-DAP hydrogel was higher (74\%) than that from both CMC-NP hydrogels. This behaviour is consistent with the higher water uptake of CMC-DAP in comparison to the two hybrid hydrogels (Figure 4).

\subsection{Release Behaviour of the Hybrid Hydrogels under the Magnetic Fields (SMF and AMF)}

The release rate of MB was also measured in the presence of static and alternating magnetic fields. In the case of the SMF the samples were subjected to a magnetic induction of 0.5 T. CMC-NP-50 released 17\% less MB (after 2 hours) under SMF than it did without SMF (Figure 6(a)). A slightly higher difference (21\%) was found for CMC-NP-70 (see Figure 6(b)).

Furthermore, two different alternating magnetic field (AMF) values were used: a magnetic induction of $0.5 \mathrm{~T}$ with a frequency of $4 \mathrm{~Hz}$, and a magnetic induction of $2 \mathrm{mT}$ with a frequency of $40 \mathrm{kHz}$.

When subjected to a magnetic induction of $0.5 \mathrm{~T}$ and a frequency of $4 \mathrm{~Hz}$, CMC-NP-50 and CMC-NP-70 show a greater $\mathrm{MB}$ release than in the absence of a magnetic field. In particular, the amount of MB released from CMC-NP-50 upon AMF is 26\% higher than that for the same hydrogel in the absence of a MF (Figure 7(a)). With CMC-NP-70 the difference is only 10\% (Figure 7(b)), showing that the greater number of nanoparticles and their aggregation (Figure 3) represent an obstacle to drug release.

The same hydrogels were also subjected to a $2 \mathrm{mT}$ magnetic field (250 times lower than the previous one), at a frequency of $40 \mathrm{KHz}(10,000$ times higher). The CMC-NP-50 shows a slightly greater release (8\%) than in the absence of AMF (Figure 8(a)), and continues to respond better than CMC-NP-70, for which the release is equal to that of the same gel without AMF (Figure 8(b)).

\section{MF-Modulated Release}

The two magnetic fields-SMF and AMF were used in succession to achieve the modulation of MB release from the CMC-NP-50 hydrogel over time, as this hydrogel shows better release behaviour in the presence of magnetic fields.

Figure 9 shows the release trend of MB from the hydrogel under the application of SMF and AMF in sequence, over 21 hours. The zero value on the vertical axis corresponds to the value of MB released from the same hydrogel in the absence of any magnetic field. The response to variations in the magnetic field was instantaneous and an "up and down" trend was observed every time the magnetic field was applied. 

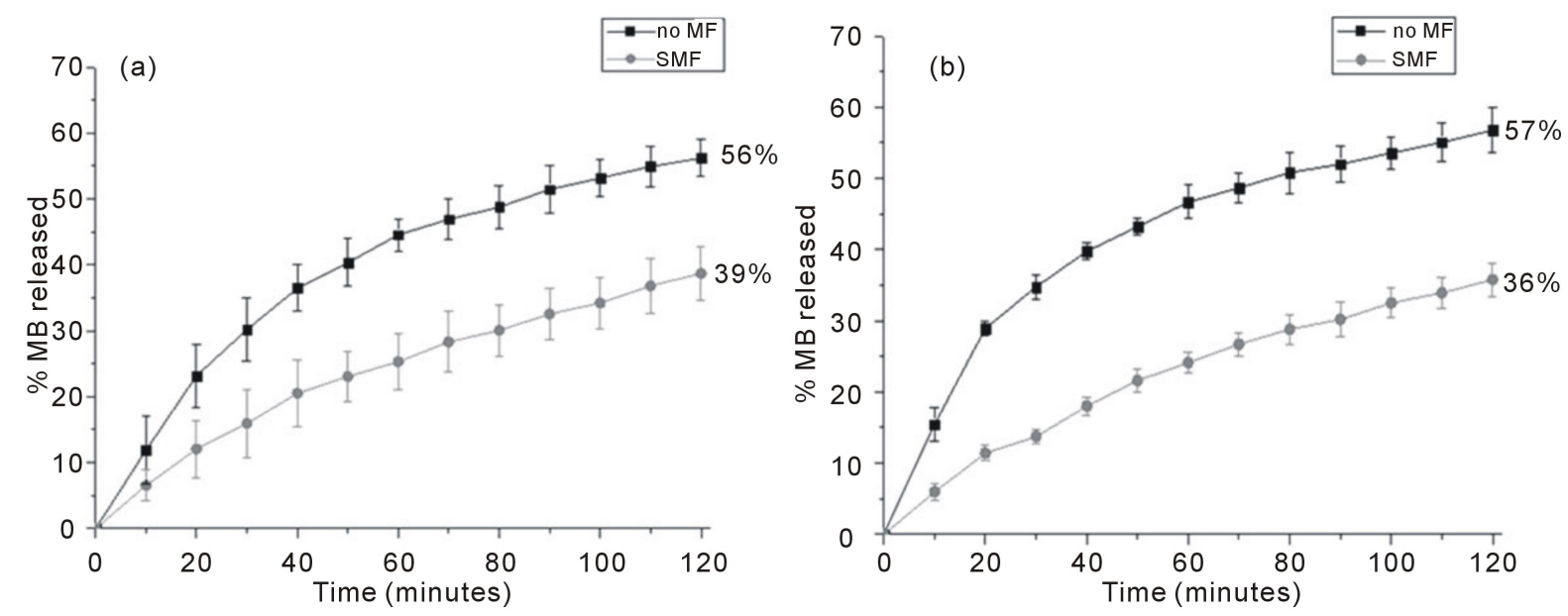

Figure 6. Release curve of methylene blue from CMC-NP-50 (A) and CMC-NP-70 (B): black squares, without SMF; grey circles, under SMF (0.5 T).
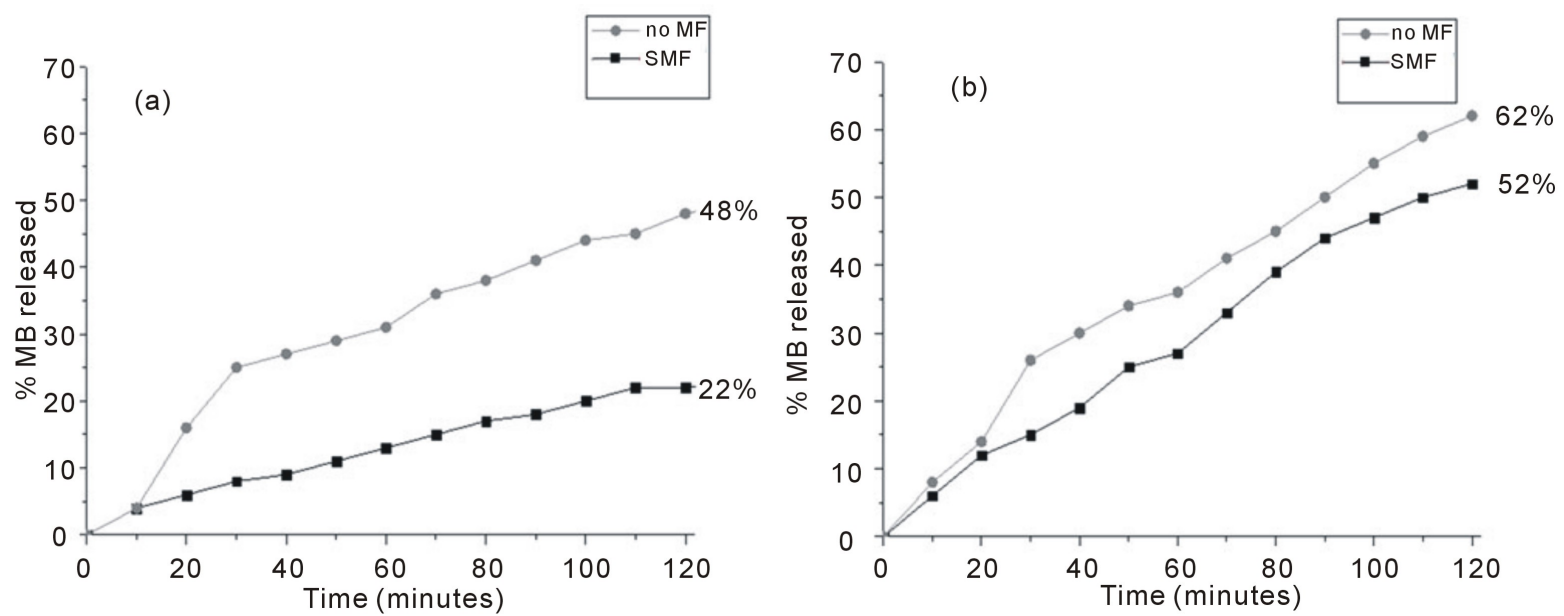

Figure 7. Release curve of methylene blue from CMC-NP-50 (A) and CMC-NP-70 (B): black squares, under AMF (4 Hz, $0.5 \mathrm{~T}$ ); grey circles, without AMF.
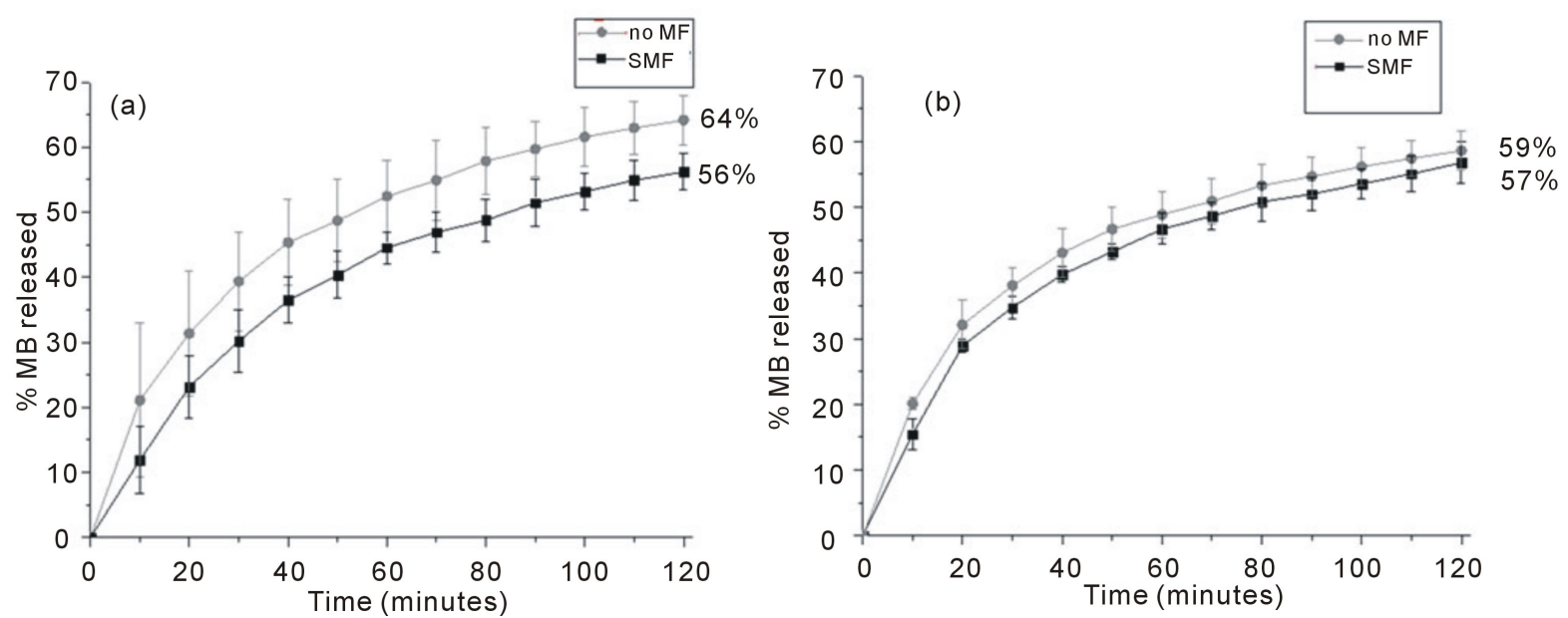

Figure 8. Release curve of methylene blue from the MB-loaded hydrogels CMC-NP-50 (A) and CMC-NP-70 (B): black squares, under $\mathrm{AMF}(40 \mathrm{KHz}, 2 \mathrm{mT})$; grey circles, without AMF. 


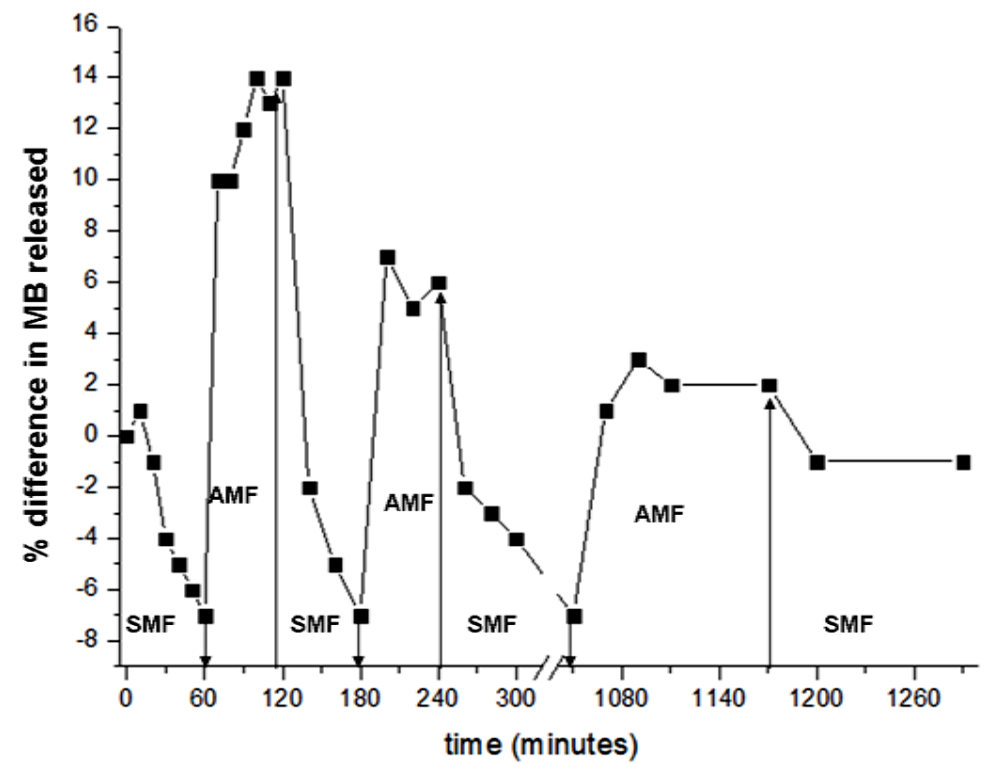

Figure 9. Release curve of methylene blue from CMC-NP-50 in $0.15 \mathrm{M} \mathrm{NaCl}$ at room temperature, under the SMF $(0.5 \mathrm{~T})$ and AMF $(0.5 \mathrm{~T}, 4 \mathrm{~Hz})$, applied in sequence. The $\mathrm{NaCl}$ solution was refreshed at every change of the magnetic field.

\subsection{Discussion}

A similar trend to that observed for the CMC-NP hydrogels submitted to the two different magnetic fields (SMF and AMF) was obtained when measuring the water uptake of the hydrogels under SMF and AMF. After about 240 minutes a plateau was reached and the WU value of both the hydrogels under AMF was always larger than in the absence of any MF, which, in turn, was greater than that under SMF, i.e. WU AMF $>$ WU no MF $>$ WU SMF (Figure 10).

During exposure to the SMF, the hydrogels were placed between two opposing permanent magnets. Elongation of the hydrogel towards the two magnets was observed (Figure 11). The hydrogels became thinner and longer under the SMF. Consequently the dimension of the pores must decrease, hindering release of MB [14] [15]. This deformation in the structure of the hydrogel is made easier as the material is softer and the G' value of CMC-NP-70 is lower than that of CMC-NP-50.

When an alternating magnetic field is applied, the different size of the nanoparticles influences the structure of the gel. In fact, the change in polarity from north to south is followed differently by the nanoparticles depending on their state of aggregation, and then their size. Small ones follow the variations in the magnetic field more easily while, as the size of the nanoparticles gradually increases, so does their difficulty in following the change of polarity. This means that larger particles break more to fit the oscillation of the magnetic field. All the nanoparticles are linked within the network of the gel, therefore the presence of nanoparticles of different sizes causes winding of the polymer chains, which in turn determines the formation of more open structures, and then pores of larger dimensions. All this is clearly represented by Figure 12, in which a chain is held fixed at both ends. When one end of the chain rotates, the chain is deformed, giving rise to loops in the chain.

All this means that the release of any molecule from a hydrogel containing magnetic NPs, under a either a static or an alternating magnetic field, depends on the structural modifications occurring in the hydrogel. A static magnetic field reduces the dimensions of the pores in the hydrogel network, hindering the release of the compound, while the presence of an alternating magnetic field increases the dimensions of the pores, allowing easier release of the drug.

This hypothesis is supported by FESEM images obtained by using the cryo-stage at $-25^{\circ} \mathrm{C}$. The surface of CMC-NP hydrogel without any magnetic fields appears smooth without any particular features as the appearance of any CMC hydrogels (see Figure 13(A)). The same hydrogel under a SMF shows a more packed structure without any pores or lamellas, but with some rough protuberances (see Figure 13(B)). On the contrary, the pre- 


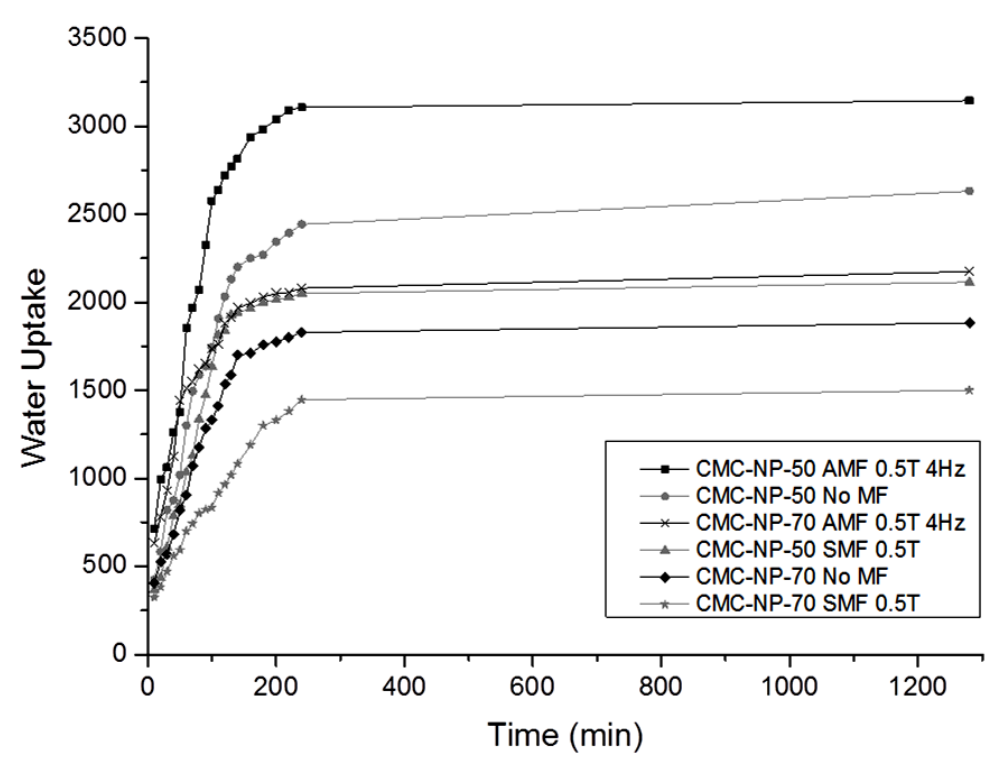

Figure 10. Kinetics of water uptake for CMC-NP hydrogels with and without magnetic fields.
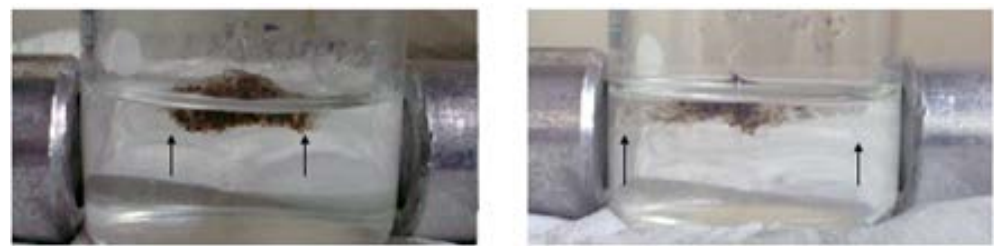

Figure 11. CMC-NP hydrogel between two opposing permanent magnets.

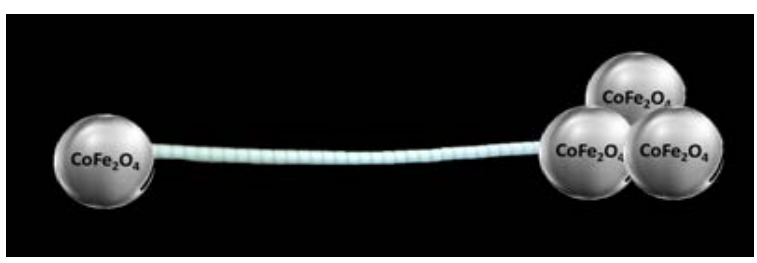

(a)

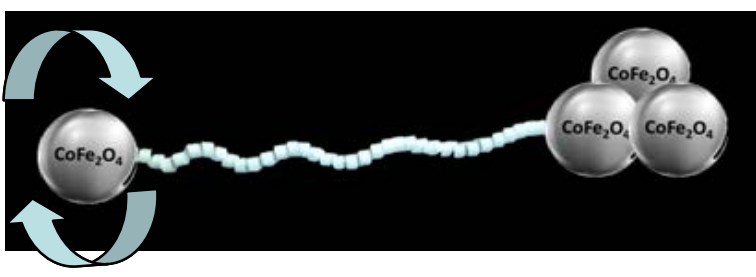

(b)

Figure 12. Schematic drawing of a polymer chain with crosslinked nanoparticles without application of AMF (a) and with application of AMF (b).

sence of AMF leads to the formation of some pores and unravelings on the hydrogel surface (see Figure 13(C)).

\section{Conclusions}

In summary, CMC hybrid hydrogels containing $\mathrm{CoFe}_{2} \mathrm{O}_{4}$ NPs are capable of responding to alternating (AMF) and static (SMF) magnetic fields, which influence their capacity to release a model drug such as MB in different ways. An AMF induces a higher release of MB than that occurs in the absence of a MF, especially at low frequency and high magnetic intensity. On the contrary, when the hybrid hydrogels are exposed to SMF, the release of MB is slowed down.

Different amounts of magnetic nanoparticles induce different responses to the two types of MF. The greater number of nanoparticles in CMC-NP-70 leads to their partial aggregation and the formation of NP clusters in the hydrogel. This creates an obstacle to drug release, thus the hybrid hydrogel containing a lower amount of nanoparticles (CMC-NP-50) shows a higher release of MB. The release behaviour is correlated with the water uptake (WU) of the two hydrogels when exposed to the same magnetic fields. The trend observed closely follows that 

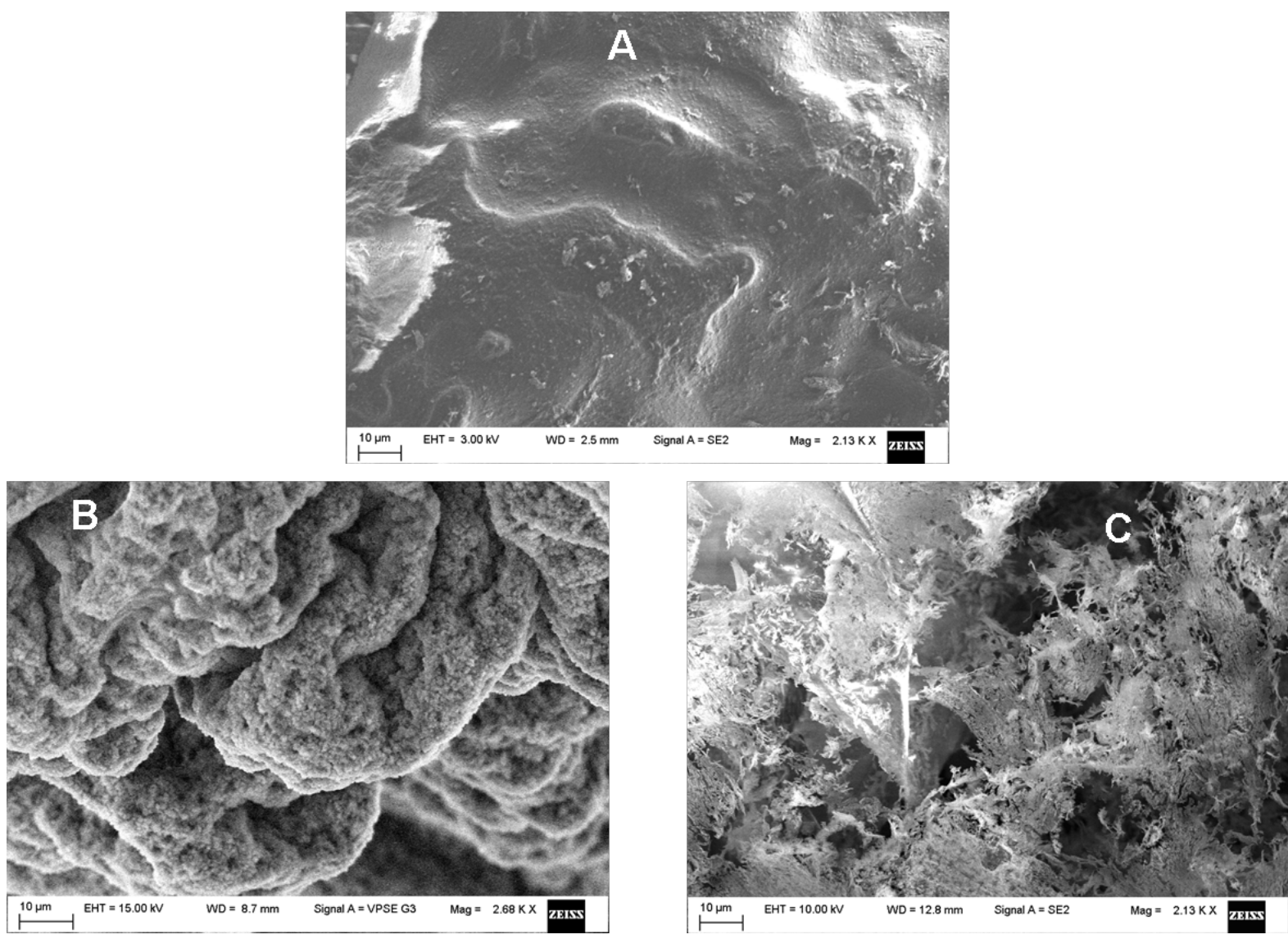

Figure 13. FE-SEM images of freeze-dried CMC-NP-50 without application of magnetic field (A), with application of SMF (B) and AMF (C).

for the drug release, i.e. $\mathrm{WU}(\mathrm{AMF})>\mathrm{WU}($ no $\mathrm{MF})>\mathrm{WU}(\mathrm{SMF})$. This explains the structural modification of the polymeric chains that occurs when the hybrid hydrogels are exposed to a MF. The static magnetic field determines a lengthening and thinning of the material, with a consequent reduction in the pore diameters. In contrast, the alternating magnetic field determines the formation of more open structures.

\section{Acknowledgements}

The authors thank the M.I.U.R. (Italian Ministry for Education, University and Research) for financial support for the project "Strategies for engineering ferromagnetic nanoparticles as crosslinkers of polymer chains: application as a targeted drug delivery system in primary and secondary bone tumors", under F.I.R.B. project RBAP11ZJFA, 2010.

\section{References}

[1] De Paoli, V.M., De Paoli, L.S.H., Spinu, L., Ingber, B., Rosenzweig, Z. and Rosenzweig, N. (2006) Effect of Oscillating Magnetic Fields on the Release Properties of Magnetic Collagen Gels. Langmuir, 22, 5894-5899. http://dx.doi.org/10.1021/la060280h

[2] Yi, J.Z. and Zhang, L.M. (2007) Biodegradable Blend Films Based on Two Polysaccharide Derivatives and Their Use as Ibuprofen-Releasing Matrices. Journal of Applied. Polymer Science, 103, 3553-3559. http://dx.doi.org/10.1002/app.24566

[3] Satarkar, N.S. and Hilt, J.Z. (2008) Nanocomposite Hydrogels as Remote Controlled Drug Delivery Systems. Acta Biomateralia, 4, 11-16. http://dx.doi.org/10.1016/j.actbio.2007.07.009

[4] Meenach, S.A., Hilt, J.Z. and Anderson, K.W. (2010) Poly(ethylene glycol)-Based Magnetic Hydrogel Nanocomposites for Hyperthermia Cancer Therapy. Acta Biomaterialia, 6, 1039-1046. 
http://dx.doi.org/10.1016/j.actbio.2009.10.017

[5] Liu, H., Wang, C., Gao, Q., Chen, J., Ren, B., Liu, X. and Tong, Z. (2009) Facile Fabrication of Well-Defined Hydrogel Beads with Magnetic Nanocomposite Shells. International Journal of Pharmaceutics, 376, 92-98. http://dx.doi.org/10.1016/j.ijpharm.2009.04.031

[6] Gaihre, B., Seob Khil, M., Rae Lee, D. and Yong Kim, H. (2009) Gelatin-Coated Magnetic Iron Oxide Nanoparticles as Carrier System: Drug Loading and in Vitro Drug Release Study. International Journal of Pharmaceutics, 365, 180189. http://dx.doi.org/10.1016/j.ijpharm.2008.08.020

[7] Meenach, S.A., Otu, C.G., Anderson, K.W. and Hilt, Z. (2012) Controlled Synergistic Delivery of Paclitaxel and Heat from Poly( $\beta$-amino Ester)/Iron Oxide-Based Hydrogel Nanocomposites. International Journal of Pharmaceutics, 427, 177-184. http://dx.doi.org/10.1016/j.ijpharm.2012.01.052

[8] Galicia, J.A., Cousin, F., Dubois, E., Sandre, O., Cabuil, V. and Perzynski, R. (2009) Static and Dynamic Structural Probing of Swollen Polyacrylamide Ferrogels. Soft Matter, 5, 2614-2624.

[9] Barbucci, R., Pasqui, D., Giani G., De Cagna, M., Fini, M., Giardino, R. and Atrei, A. (2011) A Novel Strategy for Engineering Hydrogels with Ferromagneticnanoparticles as Crosslinkers of the Polymer Chains. Potential Applications as a Targeted Drug Delivery System. Soft Matter, 7, 5558-5565. http://dx.doi.org/10.1039/c1sm05174a

[10] Barbucci, R., Giani, G., Fedi, S., Bottari, S. and Casolaro, M. (2012) Biohydrogels with Magnetic Nanoparticles as Crosslinker: Characteristics and Potential Use for Controlled Antitumor Drug-Delivery. Acta Biomaterialia, 8, 42444252. http://dx.doi.org/10.1016/j.actbio.2012.09.006

[11] Giani, G., Fedi, S. and Barbucci, R. (2012) Hybrid Magnetic Hydrogel: A Potential System for Controlled Drug Delivery by Means of Alternating Magnetic Fields. Polymers, 4, 1157-1169. http://dx.doi.org/10.3390/polym4021157

[12] Pasqui, D., De Cagna, M. and Barbucci, R. (2012) Polysaccharide-Based Hydrogels: The Key Role of Water in Affecting Mechanical Properties. Polymers, 4, 1517-1534. http://dx.doi.org/10.3390/polym4031517

[13] Tang, Y.F., Du, Y.M., Hu, X.W., Shi, X.W. and Kennedy, J.F. (2007) Rheological Characterisation of a Novel Thermosensitive Chitosan/Poly(vinyl alcohol) Blend Hydrogel. Carbohydrate Polymers, 67, 491-499. http://dx.doi.org/10.1016/j.carbpol.2006.06.015

[14] Huang, L.Y. and Yang, M.C. (2007) Behaviors of Controlled Drug Release of Magnetic-Gelatin Hydrogel Coated Stainless Steel for Drug-Eluting-Stents Application. Journal of Magnetism and Magnetic Materials, 310, 2874-2876. http://dx.doi.org/10.1016/j.jmmm.2006.11.151

[15] Liu, T.Y., Hu, S.H., Liu, K.H., Liu, D.M. and Chen, S.Y. (2006) Preparation and Characterization of Smart Magnetic Hydrogels and Its Use for Drug Release. Journal of Magnetism and Magnetic Materials, 304, e397-e399.

http://dx.doi.org/10.1016/j.jmmm.2006.01.203 